María del Carmen Bejarano Gómez',*

Rosana Braojos Bautista'

María Rosa Díez García

María del Carmen Prieto Sánchez ${ }^{2}$

Sandra Sánchez Díez ${ }^{3}$

1. Enfermera. Complejo Hospitalario de Toledo. Toledo. España.

2. Enfermera. Residencia de Mayores Benquerencia. Toledo. España.

3. Enfermera.

*Autor para correspondencia

Correo electrónico: gadea310@hotmail.com (M. ${ }^{a}$ del Carmen Bejarano Gómez).

\section{El sexo en la vejez visto desde el resto de la sociedad}

Recibido el 13 de noviembre de 2019; aceptado el 2 de marzo de 2020.

\section{RESUMEN}

Introducción: De acuerdo con la definición de sexualidad según la Organización Mundial de la Salud, el sexo atañe al ser humano durante toda su vida; sin embargo, parece que la sociedad fracciona a la persona en edades y priva de una realidad justa en el disfrute de la sexualidad en la senectud. El aumento de este sector poblacional, su esperanza de vida y mejora del estado de salud está abriendo un hueco en el interés por este tema, del que antes carecía. La mayoría de los estudios de investigación acerca de la sexualidad en la senectud cuentan como sujetos de estudio a los propios mayores; pero ¿y si estudiásemos la visión del resto de la sociedad? Objetivos: Conocer qué piensa la sociedad del sexo en la tercera edad. Metodología: Se realizó un estudio observacional descriptivo transversal de un total de 685 personas. Para la recogida de información, se realizó un cuestionario de diseńo propio, anónimo y específico que se difundió a través de

internet. Resultados: Se obtuvieron resultados que esclarecían la opinión de la sociedad mediante un análisis estadístico descriptivo.

Conclusiones: La sexualidad de las personas mayores es un tema aún con prejuicios negativos por parte de la sociedad; sin embargo, cuando a esta se le pide empatía, la respuesta es muy positiva.

PALABRAS CLAVE: Sexualidad, vejez, sociedad.

\section{ABSTRACT}

Introduction: According to the World Health Organization, sexuality concerns human beings throughout their entire lifetime. However, it seems that society categorizes people into their age groups and that the elderly are deprived of a fair reality relating to the enjoyment of sexuality in old age. The increase of this population sector, life expectancy and improvement of the state of health, is increasing interest on this subject, which was previously lacking. The majority of research studies about sexuality in old age are carried out with within the elderly sector, but what if there was a research study based on the rest of society's views on this subject? Objectives: To know what society think in relation to sex within the elderly sector. Methodology: A descriptive cross-sectional observational study of a total of 685 people was carried out. For the collection of information, an own, anonymous, and specific design questionnaire was made and disseminated through the internet. Results: Results were obtained that clarified the opinion of society through a multivariate statistical analysis with IBM SPSS Statistics 19. Conclusions: Society's views on the sexuality of the elderly is a subject with negative prejudices. However, when empathy is asked, the response is very positive.

KEYWORDS: Sexuality, elderly, society.

\section{- INTRODUCCIÓN}

La Organización Mundial de la Salud (OMS) define sexualidad como "un aspecto central del ser humano presente a lo largo de su vida. Abarca el sexo, las identidades y los papeles de género, la orientación sexual, el erotismo, el placer, la intimidad y la reproducción. La sexualidad se vivencia y se expresa a través de pensamientos, fantasías, deseos y creencias, actitudes, valores, conductas prácticas, papeles y relaciones interpersonales. La sexualidad puede incluir estas dimensiones; no obstante, no todas ellas se vivencian o se expresan siempre. La sexualidad está influida por la interacción de factores biológicos, psicológicos, sociales, económicos, políticos, culturales, éticos, legales, históricos y espirituales".

De acuerdo con esta definición, el sexo atañe al ser humano durante toda su vida; sin embargo, parece que la sociedad fracciona a la persona en edades y priva de una realidad justa en el disfrute de la sexualidad en la senectud ${ }^{1}$.
El sexo en muchas ocasiones se asocia a juventud y belleza ${ }^{2}$ y quizás es por eso por lo que existe la falsa creencia de que los mayores sufren una pérdida de interés hacia la sexualidad; sin embargo, hay estudios científicos, aunque no en demasía, que avalan que las personas mayores quieren seguir disfrutando de su sexualidad ${ }^{3}$, necesitan del contacto físico, el placer y la intimidad ${ }^{4}$, adaptándose a su estado de salud con la existencia o no de enfermedades crónicas y su nivel de independencia más o menos mermado. También avalan que la calidad de las relaciones sexuales mejora con el pasar de los años y centran su práctica en besos, caricias, seguido de coito y masturbación ${ }^{5}$.

Por tanto, la asexualidad en los mayores de 65 años queda desmentida ${ }^{6}$.

Sí que es cierto que algunos estudios recogen un declive en la práctica del sexo según aumenta la edad, pero no por ello deberíamos creer que los ancianos no tienen interés u obtienen satisfacción con ello ${ }^{7,8}$.

Una de las posibles causas de este descenso estaría en que algunos mayores no gozan de la intimidad y privacidad ideales para poder mantener 
relaciones sexuales, al vivir con familiares o estar institucionalizados, y en la educación y en el rol que se atribuye socialmente a los ancianos?.

El aumento de este sector poblacional, su esperanza de vida y la mejora del estado de salud ${ }^{10}$ está abriendo un hueco en el interés en la sexualidad de los mayores del que antes carecía.

Es interesante la diferencia del disfrute de la sexualidad según el género. Mayoritariamente se piensa que es el hombre el que tiene más interés por el disfrute de la sexualidad y que la mujer presta muy poca atención a este aspecto de su vida ${ }^{11}$; pero quizá sería bueno plantearse que ambos sexos lo viven de un modo distinto ${ }^{12}$.

La mayoría de los estudios de investigación acerca de la sexualidad en la senectud cuentan como sujetos de estudio a los propios mayores. ¿Y si estudiásemos la visión del resto de la sociedad?

\section{- OBJETIVO}

Conocer la opinión de la sociedad acerca de la sexualidad en la tercera edad.

\section{- METODOLOGÍA}

Se realizó un estudio observacional descriptivo transversal tomando como población a estudio aquellas personas de entre 18 y 65 años que vivieran en el territorio español. El tamaño muestral fue de 685 personas que participaron en la encuesta que se realizó entre septiembre de 2017 y enero de 2018.

Para la recogida de información, se realizó un cuestionario de diseño propio, anónimo y específico. Dicho cuestionario cuenta con 17 ítems, de los cuales 2 , la edad y procedencia, serían variables independientes (Anexo 1).

El cuestionario creado se difundió a través de internet mediante correo electrónico, mensajería instantánea y redes sociales, pudiendo de esta manera llegar a todo el territorio nacional y facilitando así la accesibilidad a toda la sociedad interesada en participar.

Los resultados se obtuvieron mediante un análisis descriptivo simple.

El estudio cuenta con limitaciones, ya que no es un cuestionario validado, pero la falta de uno que recoja el tema a estudio nos lleva a su creación y posible inicio de posteriores trabajos y conclusiones acerca de la opinión del resto de la sociedad sobre el sexo en la tercera edad, basándonos en la falta de bibliografía al respecto.

También cabe decir que el cuestionario permitía, en algunas preguntas, responder no sabe/no contesta, ya que quizá algunas personas no tendrían una opinión clara al respecto. Esto podría sesgar un poco los porcentajes totales.

Además, los datos recogidos proceden en su gran mayoría de Castilla-La Mancha y la Comunidad de Madrid, ya que la difusión fue mayor debido a la procedencia de las investigadoras.

\section{- RESULTADOS}

La población encuestada tiene una edad comprendida entre los 18 y 65 años (fig. 1) y proceden de todo el territorio nacional (fig. 2).

Los resultados de las distintas preguntas realizadas en la encuesta se presentan a continuación:

- ¿Cree que los mayores de 65 años conservan el interés por la sexualidad? El $80,6 \%$ opinó que sí, el $12,7 \%$ que no y el $6,7 \%$ no sabe o no contesta (NS/NC).

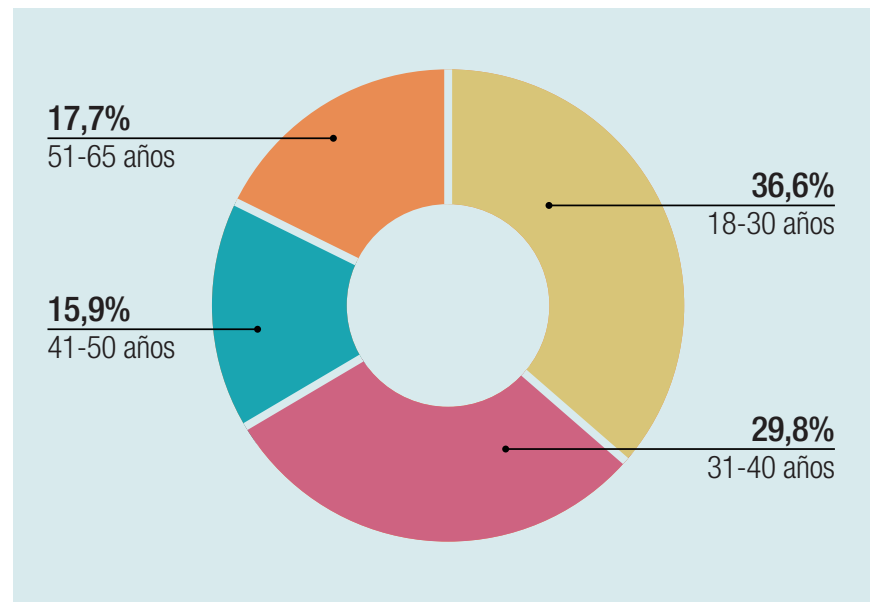

Figura 1. Edad de la población encuestada.

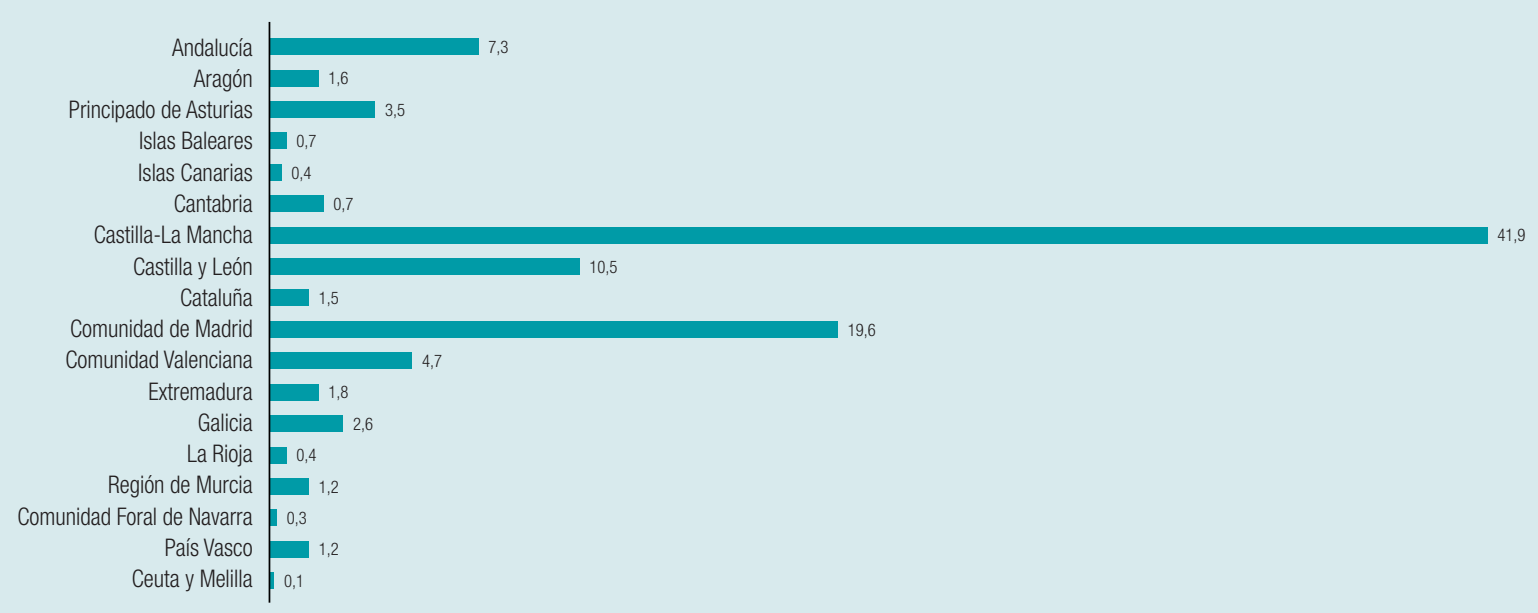

Figura 2. Procedencia de los encuestados. 
- ¿Quién cree que presta más atención a su sexualidad en los mayores de 65 ańos? El 59\% respondió que los hombres, el 7\% que las mujeres, el 30,1\% que ambos sexos y el 3,9\% NS/NC.

- ¿Cómo considera el disfrute de la sexualidad en los mayores de 65 años? El 56,8\% opinó que bien, el 3,2\% que mal, el 29,5\% que normal, el $5,3 \%$ que si no se habla de ello mejor, y otro 5,3 NS/NC.

- Cree que dicho grupo de población cuenta con la intimidad necesaria para el disfrute de su sexualidad? El 56,6\% dijo que sí, el $35 \%$ que no y el $8,3 \%$ NS/NC.

- ¿Cree que las experiencias sexuales en los mayores de 65 ańos pueden ser igual o más placenteras que antes? El 60,4\% opinó que sí, el $21,9 \%$ que no y el $17,7 \%$ NS/NC.

- ¿El envejecimiento y/o la enfermedad cree que son motivos para abandonar el disfrute de la sexualidad? El 65,4\% contestó que no, el $30,5 \%$ que sí y el $4,1 \%$ NS/NC.

- ¿Cree que los mayores de 65 años tienen respaldo de la sociedad para el disfrute de su sexualidad, sin prejuicios? (fig. 3).

- ¿La publicidad de artículos para el disfrute de la sexualidad cree que va también dirigida a este colectivo? El 80\% opinó que no, el 15,5\% que sí y el 4,5\% NS/NC.

- ¿Qué tipo de productos cree que dirige la publicidad para este grupo de población? El 75,6\% respondió que medicamentos, el 8,2\% que alimentación, el $8 \%$ que productos de belleza, el 3,1\% que sexualidad y el $5,1 \%$ que otros.



Figura 3. Respaldo de la sociedad a los mayores de 65 años para el disfrute de su sexualidad sin prejuicios.

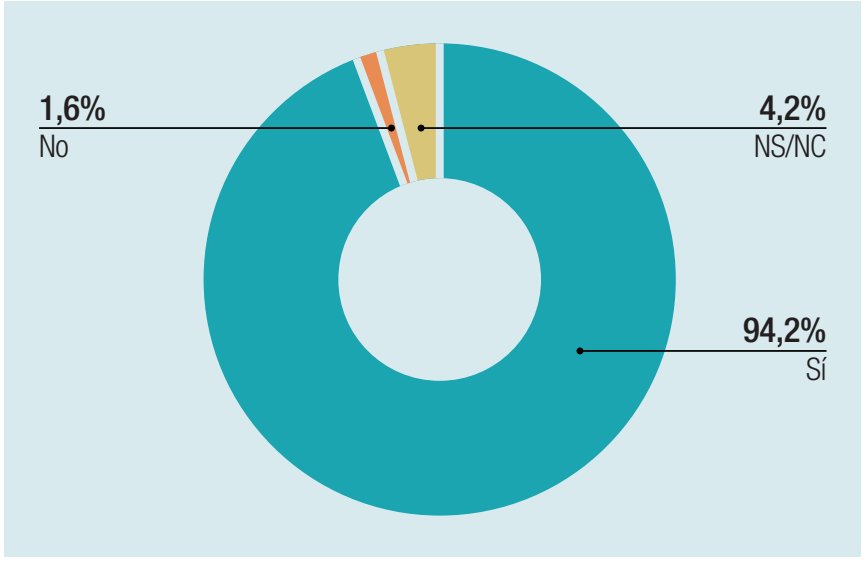

Figura 4. Práctica del sexo, buena para la salud en mayores de 65 años.
- Si sorprendiera a una persona mayor de 65 años practicando sexo... ¿qué le produciría? El 56,4\% opinó que aceptación, el 19,6\% vergüenza, el 2,6\% asco, el 0,9\% rechazo y el 20,6\% otros.

- ¿Le produciría la misma sensación que si sorprendiera a alguien joven? un $69,5 \%$ contestó que sí, un $25,7 \%$ que no y un 4,8\% NS/ NC.

- ¿Cree que una persona mayor de 65 años que enviuda debería dejar de disfrutar de su sexualidad? El 93\% contestó que no, el 3,9\% que sí y el 3,1\% NS/NC.

- ¿Cree que la práctica del sexo puede ser buena para la salud en los mayores de 65 años? (fig. 4).

- Viagra ${ }^{\oplus}$ geles para combatir la sequedad vaginal... ¿cuál cree que está más difundido y/o socialmente mejor visto? (fig. 5).

- Cuando usted sea mayor de 65 años... ¿se privará del disfrute de la sexualidad? El 86,3\% dijo que no, el 1,2\% que sí y el 12,6\% NS/NC.

\section{- DISCUSIÓN}

La sexualidad de las personas mayores es un tema del que quizá no se habla en demasía vista la escasa bibliografía en cuanto a estudios científicos al respecto, ya que puede considerarse un tabú e incluso algo negativo para algunos miembros de la sociedad ${ }^{13}$.

Algo que se ve si utilizamos la publicidad como el espejo de la sociedad, pero maquillada con influencia platónica, donde no se valora que los artículos para el disfrute de la sexualidad vayan destinados a ese colectivo y sí los medicamentos, la alimentación o los productos de belleza... dibujándonos una persona mayor tipo envejecida, preocupada por su salud y apariencia, pero de la que no se contemplan todos los aspectos de su persona como es el de la sexualidad ${ }^{14,15}$.

En cuanto a género se refiere, la opinión de que el hombre presta más atención a su sexualidad se corresponde con los estudios contrastados que tenían a este sector poblacional como muestra a estudio, pero se abre una puerta al cambio que, aunque no ve a la mujer como más interesada, equilibra a ambos sexos en el interés por este aspecto de su vida. Los mayores disfrutan de su sexualidad y no solo la reducen a la genitalidad $^{16}$.

Este trabajo abre sin dudas nuevas líneas de investigación, donde conozcamos los distintos modos de disfrutar el sexo en personas mayores de 65 años, donde podamos descubrir las diferencias existentes en el modo de vivirla entre sexos, comparando las opiniones del resto de la sociedad con las propias del colectivo.



Figura 5. Producto para la sexualidad más difundido y/o socialmente mejor visto. 


\section{- CONCLUSIONES}

Según este estudio, la sociedad cree que los mayores conservan el interés por su sexualidad valorándolo como algo normal, positivo.

Mayoritariamente, opinan que es el hombre el que tiene más interés por el disfrute de su sexualidad y que la mujer presta muy poca atención a este aspecto de su vida. Sí es cierto que también parte de esta sociedad piensa que ambos sexos prestan atención a su sexualidad.

En cuanto a la intimidad necesaria para el disfrute de la sexualidad por parte de los ancianos, la mayoría de la sociedad encuestada piensa que sí que gozan de ella, a pesar de que en muchas ocasiones viven institucionalizados, en casa de un familiar, etc.

El placer obtenido en los mayores de 65 ańos es igual o superior a pesar del envejecimiento y/o la edad a juicio de los encuestados y el estado civil no debe influir en la sexualidad de la persona mayor.

A pesar de creer que los mayores conservan el interés por disfrutar su sexualidad y otorgarles su aprobación, se sigue pensado que la sociedad en general no les da el respaldo necesario para gozar de ella sin prejuicios.
En el caso de sorprender a una persona mayor de 65 ańos practicando sexo, la sensación provocada en su mayoría es de aceptación, sin diferencia significativa con respecto a descubrir a una persona joven en la misma situación.

El sexo es bueno para la salud según la mayoría encuestada, a pesar de que, desde ámbitos como el de la publicidad, los productos mayoritarios que ofrecen a este sector sean de alimentos, medicamentos, etc. y no productos relacionados con la sexualidad.

En cuanto a estos productos relacionados con la sexualidad, como el caso de la Viagra y los geles para la sequedad vaginal, parece que ambos productos son igualmente conocidos y aceptados socialmente sin importar a que género van destinados.

Los encuestados no quieren privarse de disfrutar su sexualidad cuando sean mayores de 65 ańos, mostrando una empatía con los mayores que no aísla a este segmento poblacional

\section{Conflicto de intereses}

Las autoras declaran no tener ningún conflicto de intereses.

\section{Anexo 1. Encuesta realizada}

El sexo en la tercera edad visto desde el resto de la sociedad, ¿un tabú? Esta encuesta es anónima y realizada por enfermeras para un trabajo de investigación; son solo unos minutos y su opinión nos será muy útil, agradecemos su colaboración.

*Obligatorio

1. ¿Qué edad tiene?*Marque solo un círculo.

$\bigcirc$ Entre 18 y 30 años

$\bigcirc$ Entre 31 y 40 años

Entre 41 y 50 años

Entre 51 y 65 años

2. ¿Dónde vive?* Marque solo un círculo

Andalucía

Aragón

Principado de Asturias

Islas Baleares

Canarias

Cantabria

Castilla-La Mancha

Castilla y León

Cataluña

$\bigcirc$ Com. Valenciana

Extremadura

Galicia

La Rioja

Com. Madrid

Región de Murcia

Com. Foral de Navarra

País Vasco

$\bigcirc$ Ceuta o Melilla

3. ¿Cree que los mayores de 65 años conservan el interés por la sexualidad? Marque solo un círculo.

O Sí

No

$\mathrm{NS} / \mathrm{NC}$
4. ¿Quién cree que presta más atención a su sexualidad en los mayores de 65 años? Marque solo un círculo.
$\bigcirc$ Hombres
Mujeres
Ambos
$\mathrm{NS} / \mathrm{NC}$

5. ¿Cómo considera el disfrute de la sexualidad en los mayores de 65 años? Marque solo un círculo.
$\bigcirc$ Bien
Mal
Normal
Si no se habla de ello, mejor
NS/NC

6. ¿Cree que dicho grupo de población cuenta con la intimidad necesaria para el disfrute de su sexualidad?* Marque solo un círculo.

O Sí

$\mathrm{O}$ No

$\mathrm{NS} / \mathrm{NC}$

7. ¿Cree que las experiencias sexuales en los mayores de 65 años pueden ser igual o más placenteras que antes?* Marque solo un círculo.
Sí
No
$\mathrm{ONS} / \mathrm{NC}$

8. El envejecimiento y /o la enfermedad ¿cree que son motivos para abandonar el disfrute de la sexualidad? * Marque solo un círculo.

Sí

No

$\mathrm{ONS} / \mathrm{NC}$

9. ¿Cree que los mayores de 65 años tienen respaldo de la sociedad para el disfrute de su sexualidad, sin prejuicios? * Marque solo un círculo.

Sí

No

$\mathrm{NS} / \mathrm{NC}$

10. ¿La publicidad de artículos para el disfrute de la sexualidad cree que va también dirigida a este colectivo?* Marque solo un círculo.
Sí
No
$\mathrm{NS} / \mathrm{NC}$

11. ¿Qué tipo de productos cree que dirige la publicidad para este grupo de población?* Marque solo un círculo.

Alimentación

Medicamentos

$\bigcirc$ Productos de belleza

Sexualidad

Otros 


\section{Anexo 1. Encuesta realizada (cont.)}

12. Si sorprendiera a una persona mayor de 65 años practicando sexo... ¿qué le produciría?* Marque solo un círculo.

Rechazo

Vergüenza

Asco

Aceptación

Otros

13. ¿Le produciría la misma sensación que si sorprendiera a alguien joven?* Marque solo un círculo.
O Sí
No
$\mathrm{NS} / \mathrm{NC}$

14. ¿Cree que una persona mayor de 65 años que enviuda debería dejar de disfrutar de su sexualidad? * Marque solo un círculo.
Sí
No
$\mathrm{NS} / \mathrm{NC}$

15. ¿Cree que la práctica del sexo puede ser buena para la salud en los mayores de 65 años? Marque solo un círculo.
Sí
No
$\mathrm{ONS} / \mathrm{NC}$
S Sí
O no
$\mathrm{NS} / \mathrm{NC}$

16. Viagra ${ }^{\circledR}$, geles para combatir la sequedad vaginal... ¿Cuál cree que está más difundido y/o socialmente mejor visto? Marque solo un círculo.
O Sí
No
$\mathrm{ONS} / \mathrm{NC}$

17. Cuando usted sea mayor de 65 años... ¿se privará del disfrute de la sexualidad? Marque solo un círculo.

\section{BIBLIOGRAFÍA}

1. Federación Española de Sociedades de Sexología. Estudio sobre las actitudes y los hábitos sexuales en España. Santander: VII Congreso Español de Sexología, 2004

2. Figuera L. Sexualidad en los ancianos. Rol de Enfermería. 1990;145:39-46.

3. Nieto JA. Los ancianos españoles y su sexualidad. En: Nieto JA, ed. La sexualidad de las personas mayores en España. Madrid: Instituto Nacional de Servicios Sociales; 1995.

4. López F, Olazábal JC. Sexualidad en la vejez. Madrid: Pirámide;1998

5. 5. López F Sexualidad y afectos en la vejez. Madrid: Pirámide; 2012

6. Corominas Clemente C, García Blázquez M, Ortega Martínez MD Serna Roldán C. Sexualidad y Tercera Edad. Revista de Enfermería 1995;5:6-13.

7. Kaiser F. Sexuality in the elderly. Urol Clin N Am. 1996;23(1):99-109.
8. Pulido L. El sexo en la vejez. El Heraldo.co [Internet] 13 Ago 2017 Colombia. Disponible en: https://www.elheraldo.co/entretenimiento/el-sexo-en-la-tercera-edad-392195

9. CSIC. "Un perfil de las mayores en España 2019" (acceso 28 de enero de 2020). Disponible en: http://envejecimientoenred.es/un-perfil-de-las-personas-mayores-en-espana-2019-indicadores-estadisticos-basicos/

10. Ministerio de Sanidad y Política social. Resultados de la encuesta nacional de salud sexual. Informe No. 57404. 2009. Recuperado de http://www.imsersomayores.csic.es

11. Palacios D. Sexual behaviors among older adults in Spain: results from a population-based national sexual health survey. J Sex Med. 2012;9(1):121-9

12. Master W, Jhonson V. La respuesta sexual humana. Buenos Aires: Interamericana; 1967.
13. Cobas Moreira Y. "La sexualidad en el adulto mayor desde la perspectiva psicosocial" (acceso 27 de febrero de 2020). Disponible en: http://www.eumed.net/rev/cccss/16/ymc.html

14. Ramos Soler I, Carretón-Ballester MC. "Presencia y representación de las personas mayores en la publicidad televisiva: el caso español". Rev Esp Geriatr Gerontol. 2012;47(2):55-61.

15. López V. "La publicidad y la tercera edad, un segmento olvidado" (acceso 27 de febrero de 2020). Disponible en: https://www. marketingdirecto.com/punto-de-vista/la-columna/la-publicidad-y-la-tercera-edad-un-segmento-olvidado-vicente-lopez

16. Leyva-Moral JM. "La expresión sexual de los ancianos. Una sobredosis de falsos mitos". Index Enferm. 2008;17(2). (acceso 29 de enero de 2020) Disponible en: https://scielo.isciii.es/scielo.php?script=sci_arttext\&pid=S1132-12962008000200010 